DOI https://doi.org/10.18551/rjoas.2020-11.19

\title{
THE RELATIONSHIP BETWEEN ECONOMIC GROWTH AND AGRICULTURAL LAND-USE INTENSITY
}

\author{
Wei Zhu \\ ORCID: 0000-0001-7822-0095 \\ Ruimei Wang* \\ ORCID: 0000-0001-9964-9421 \\ College of Economics and Management, China Agricultural University, Beijing, China \\ *E-mail: wangruimei@cau.edu.cn
}

\begin{abstract}
This study aims to investigate the relationship between economic growth and agricultural land-use intensity for 86 countries over the period 1990-2017. Specifically, this study analyzes the relationship between economic growth and fertilizer use intensity, pesticide use intensity, cropping frequency, production value of crops per area, GHG emissions per area. The results suggest the existence of an inverted-U-shaped relationship between economic growth and each of the agricultural land-use intensity indicators, which is consistent with the Environmental Kuznets Curve (EKC) hypothesis. This implies that in the early stages of economic growth, agricultural land-use intensity rises steadily as per capita income grows, and then begins to decline as per capita income continues to grow after reaching the turning point. A mediation analysis is conducted to test the potential causes behind the relationship.
\end{abstract}

\section{KEY WORDS}

Environmental Kuznets Curve, inverted-U-shaped relationship, land-use, economic growth.

The world population has grown steadily, people with higher purchasing power comes a greater demand for processed food, meat, dairy, and fish, all of which add pressure to the food supply system (Godfray et al., 2010). At the same time, more than 820 million people in the world are still hungry today, about 2 billion people in the world experience moderate or severe food insecurity (FAO et al., 2019). Feeding a growing world population may require an additional 2.7-4.9 Mha of cropland per year on average (Lambin and Meyfroidt, 2011), but there is substantially less potential additional cropland than is generally assumed once constraints and trade-offs are taken into account (Lambin et al., 2013). Intensification of agricultural land use may have the potential to satisfy the increasing demand for food (Godfray et al., 2010). However, intensification of agricultural land use can lead to ecological damage, soil degradation, environmental pollution, and create health risks to livestock and humans. For example, irrigation practices can limit the amount of water available for other uses; runoff from pesticides and fertilizers can degrade water quality; the use of agricultural chemicals can enhance the growth of invasive plants, which can contribute to decreases in biodiversity; carbon emission from agricultural land use accounts for about $9 \%$ of total carbon emissions (Cassou, 2018; EPA, 2020; FAOSTAT, 2017; Reay et al., 2012). To address these negative impacts, it is now widely recognized that agricultural land use must be sustainable, that is, the use of agricultural land at rates that do not exceed the capacity of Earth to replace them (Godfray et al., 2010; Pellegrini and Fernandez, 2018; Tilman et al., 2011).

The Environmental Kuznets Curve (EKC) is often used to analyze the relationship between economic growth and various indicators of environmental degradation. The common point of most studies is that environmental degradation increases faster than per capita income in the early stages of economic growth and slows down relative to GDP growth in higher per capita income levels (Dinda, 2004; Grossman and Krueger, 1995). This implies that there is an inverted-U-shaped relationship between economic growth and the environmental indicator (Stern, 2004). Environmental indicators used in most studies include atmospheric indicators, freshwater indicators, land indicators, oceans, seas coasts and biodiversity indicators (Sarkodie and Strezov, 2019). 
With respect to the relationship between economic growth and agricultural land-use intensity, there are some studies have separately tested the EKC hypothesis on pesticide use, fertilizer use, and cultivated area, etc. (Singh and Narayanan, 2015) observed a U-shaped relationship between fertilizer consumption per hectare and per capita income, an inverted-U-shaped relationship between pesticide consumption per hectare and per capita income, using data of the Indian states in 1990-2008. (Zhang et al., 2016) found that agricultural inputs (consumption of fertilizer, pesticide, and agricultural film) and economic growth in the Three Gorges Reservoir Area in China between 2003 and 2012 fulfill the EKC hypothesis. (RodríGuez-Meza et al., 2004) analyzed agricultural land use at the household level, obtained the evidence of an EKC relating farmed area to per capita income. (Managi, 2006) demonstrated that pesticide use followed the inverted-U-shaped curve for US agriculture in 48 states in 1970-1997. (Ghimire and Woodward, 2013) found a N-shaped relationship between the pesticide use and per capita GDP using data of 94 countries in 1990-2000. Using data of 119 countries in 1990-2009, (Schreinemachers and Tipraqsa, 2012) found that as per capita GDP increased, growth in the intensity of pesticide use diminished and then became negative, suggesting the existence of an inverted-U-shaped curve. (Hedlund et al., 2020) demonstrate a positive relationship between economic development and pesticide consumption over time, with no decline in use at higher levels of economic development. (Ali et al., 2017)'s results do not support the hypothesis of an inverted-U-shaped relationship between agricultural growth and $\mathrm{CO} 2$ emissions in the long run or the short run in case of Pakistan during 1960 to 1990 . However, the agricultural land-use intensity can be described from three dimensions including input intensity, output intensity, and human-induced but unintended outcomes (Erb et al., 2013). To the best of our knowledge, no previous research has investigated the relationship between economic growth and various agricultural land-use intensity indicators using the same data source. The current study attempts to fill this gap.

In this study, we examine data compiled by the Food and Agriculture Organization (FAO) on fertilizer use, pesticide use, cropping area, production value, and net carbon emissions for 86 countries over the period 1990-2017. This study aims to investigate the relationship between economic growth and each of the agricultural land-use intensity indicators. Furthermore, a mediation analysis is conducted to test the potential causes behind the EKC for agricultural land-use intensity.

\section{MATERIALS AND METHODS OF RESEARCH}

Data and variable definitions. The data for our analysis were extracted from FAO database (FAOSTAT, 2017). Following (Erb et al., 2013), the agricultural land-use intensity, which is the dependent variable in this study, integrates three dimensions: (a) input intensity measured by fertilizer use intensity, pesticide use intensity, and cropping frequency; (b) output intensity measured by production value of crops per area; (c) the associated system-level impacts of agricultural land-based production measured by greenhouse gas (GHG) emissions per area. The fertilizer use intensity is measured by the use of chemical and mineral fertilizers per area of cropland, which corresponds to the sum of arable land and permanent crops. The pesticide use intensity is measured by the use of pesticides per area of cropland. The cropping frequency is defined as the ratio of area harvested to area of cropland. The production value of crops per area is defined as the ratio of gross production value of crops (constant 2014-2016 million US\$) to area of cropland. The GHG emissions per area is defined as the ratio of GHG emissions to area of cropland. The GHG emissions includes emissions from crop residues, cultivation of organic soils, manure applied to soils, manure left on pasture, and synthetic fertilizers, expressed as $\mathrm{Gg} \mathrm{CO} 2$ and $\mathrm{CO} 2 \mathrm{eq}$ (from $\mathrm{CH} 4$ and $\mathrm{N} 2 \mathrm{O}$ ). Due to the availability of data, a total of 86 countries are included in this study. Although the averaging and simplifications in the data mean that the data are imperfect, we have the advantage of being able to include 86 countries with all the agricultural land-use intensity indicators for each country. As data for all years are available for all the 86 countries, the panel data are strongly balanced. The fertilizer use data are in a time series from 2002 to 
2017, the production value data are from 1990 to 2016, and the data of other indicators are from 1990 to 2017. The rationale for using panel data is to minimize unobserved heterogeneity bias and some dynamics of land use that are difficult to investigate with cross-sectional data only (Ghimire and Woodward, 2013).

The major explanatory variable of interest in this study is economic growth which is measured by per capita GDP (expressed in constant 2015 US\$). Changes in the proportions of different categories of crops will affect the agricultural land-use intensity, thus the proportions of cereals, vegetables, fruits, roots and tubers, coarse grain, and oil crops are used as control variables. The descriptive statistics are provided in Table 1. The correlation coefficients between various agricultural land-use intensity indicators are shown in Table 2.

Table 1 - Descriptive statistics

\begin{tabular}{|c|c|c|c|c|c|}
\hline Variable & Obs. & Mean & Std. Dev. & Min. & Max. \\
\hline Fertilizer use intensity (kg/ha) & 1376 & 117.726 & 99.032 & 0.745 & 579.800 \\
\hline Pesticide use intensity (kg/ha) & 2408 & 2.940 & 3.453 & 0.005 & 19.450 \\
\hline Cropping frequency & 2408 & 0.909 & 0.344 & 0.0729 & 2.661 \\
\hline Production value of crops (\$/ha) & 2322 & 1053.302 & 825.645 & 54.441 & 5046.765 \\
\hline GHG emissions (kg CO2eq/ha) & 2408 & 1650.516 & 1248.244 & 110.979 & 9229.642 \\
\hline GDP per capita $(\$)$ & 2408 & 13026.980 & 16039.740 & 172.498 & 83056.550 \\
\hline Share of cereals & 2408 & 0.451 & 0.163 & 0.003 & 0.887 \\
\hline Share of vegetables & 2408 & 0.044 & 0.077 & 0.001 & 0.709 \\
\hline Share of fruits & 2408 & 0.077 & 0.091 & 0.002 & 0.684 \\
\hline Share of roots and tubers & 2408 & 0.041 & 0.055 & 0.001 & 0.462 \\
\hline Share of coarse grain & 2408 & 0.206 & 0.119 & 0.000 & 0.460 \\
\hline Share of oil crops & 2408 & 0.111 & 0.121 & 0.000 & 0.772 \\
\hline
\end{tabular}

Table 2 - Correlation coefficients between various agricultural land-use intensity indicators

\begin{tabular}{ccccc}
\hline & $\begin{array}{c}\text { Fertilizer use } \\
\text { intensity }\end{array}$ & $\begin{array}{c}\text { Pesticide use } \\
\text { intensity }\end{array}$ & $\begin{array}{c}\text { Cropping } \\
\text { frequency }\end{array}$ & $\begin{array}{c}\text { Production value } \\
\text { of crops }\end{array}$ \\
\hline Fertilizer use intensity & 1.000 & & & \\
Pesticide use intensity & 0.616 & 1.000 & 1.000 & \\
Cropping frequency & -0.003 & -0.178 & 0.082 & 1.000 \\
Production value of crops & 0.570 & 0.543 & 0.066 & 0.500 \\
GHG emissions & 0.587 & 0.417 & & 1.000 \\
\hline
\end{tabular}

According to the method adopted in the previous literature (e.g.,(Bimonte and Stabile, 2017; Kuznets, 1955; Pontarollo and Serpieri, 2020; Sarkodie and Strezov, 2019)), this study defined the empirical model as follows:

$$
\begin{aligned}
& \ln y_{i t}=\beta_{0}+\beta_{1} \ln G D P_{i t}+\beta_{2}\left(\ln G D P_{i t}\right)^{2}+\theta_{i}+\gamma_{t}+\varepsilon_{i t} \\
& \ln y_{i t}=\beta_{0}+\beta_{1} \ln G D P_{i t}+\beta_{2}\left(\ln G D P_{i t}\right)^{2}+\sum \beta_{j} Z_{i j t}+\theta_{i}+\gamma_{t}+\varepsilon_{i t}
\end{aligned}
$$

Where: $i$ and $t$ represent country $i$ and year $t$, respectively; $y$ represents agricultural land-use intensity indicators; GDP denotes per capita GDP; $z$ represents other control variables; $\theta$ indicates the country's fixed effect; $\gamma$ indicates the year fixed effect; $\beta$ 's are the coefficient estimates of the regressors; $\varepsilon$ represents the random error term.

In a typical method for estimating the equations (1) and (2), the EKC hypothesis is valid if $\beta_{1}>0, \beta_{2}<0$. This implies that there is an inverted-U-shaped relationship between economic growth and agricultural land-use intensity. The "turning point" income, where the agricultural land-use intensity is at a maximum, is given by $\exp \left(-\beta_{1} /\left(2 \beta_{2}\right)\right)$.

\section{RESULTS OF STUDY}

In the empirical estimation this study proceeds as follows. First, the reduced form model shown in equation (1) is estimated separately for fertilizer use intensity, pesticide use intensity, cropping frequency, production value of crops per area, and GHG emissions per area. Second, the proportions of different categories of crops are controlled in the model shown in equation (2). This enables us to check for the robustness of the coefficients associated with per capita GDP and its squared term to the inclusion of additional control variables. The 
equations were estimated using random effect estimator (RE), fixed effects estimator (FE), and panel-correction standard error (PCSE) models respectively. The joint significance test of the year dummy variable indicates that the year dummy variable should be included in the model, thus a two-way fixed effects model should be adopted. Hausmann test indicates that FE model rather than RE model should be chosen. Modified Wald test for groupwise heteroskedasticity rejects the null hypothesis of homoscedasticity. Pesaran's test of cross-sectional independence cannot reject the null hypothesis that there is no cross-sectional correlation. Therefore, PCSE model rather than FE model should be chosen.

The estimation results for fertilizer use intensity are presented in Table 3 . The coefficients estimated by FE and PCSE are the same, thus only the results of RE and PCSE are shown in the table. The coefficients of per capita GDP and its squared term obtained by different estimation methods have little difference.

Table 3 - Estimation results for fertilizer use intensity

\begin{tabular}{|c|c|c|c|c|c|c|c|c|}
\hline & $\mathrm{RE}$ & PCSE & $\mathrm{RE}$ & PCSE & $\mathrm{RE}$ & PCSE & $\mathrm{RE}$ & PCSE \\
\hline \multirow[t]{2}{*}{ InGDP } & $3.469^{\star \star \star}$ & $3.693^{\star * \star}$ & $3.453^{\star \star \star}$ & $3.832^{\star * \star}$ & $3.473^{\star \star \star}$ & $3.714^{\star \star \star}$ & $3.485^{\star \star \star}$ & $3.839^{* * *}$ \\
\hline & (1.099) & $(0.407)$ & $(1.137)$ & $(0.461)$ & (1.105) & $(0.452)$ & (1.132) & $(0.478)$ \\
\hline \multirow[t]{2}{*}{$(\operatorname{lnGDP})^{2}$} & $-0.168^{\star * *}$ & $-0.177^{\star * \star}$ & $-0.172^{* * *}$ & $-0.194^{\star * *}$ & $-0.167^{* * *}$ & $-0.179^{* * *}$ & $-0.171^{* * *}$ & $-0.190^{* * *}$ \\
\hline & $(0.0617)$ & $(0.0227)$ & $(0.0633)$ & $(0.0285)$ & $(0.0618)$ & $(0.0259)$ & $(0.0628)$ & (0.0292) \\
\hline Country's fixed effect & Yes & Yes & Yes & Yes & Yes & Yes & Yes & Yes \\
\hline Year fixed effect & No & No & Yes & Yes & No & No & Yes & Yes \\
\hline \multirow[t]{2}{*}{ Share of cereals } & & & & & -2.068 & $-2.083^{* *}$ & -1.389 & $-1.328^{*}$ \\
\hline & & & & & $(1.441)$ & $(0.816)$ & $(1.320)$ & $(0.797)$ \\
\hline \multirow[t]{2}{*}{ Share of vegetables } & & & & & -0.222 & -0.846 & 0.0702 & -0.501 \\
\hline & & & & & $(1.563)$ & $(1.066)$ & $(1.477)$ & $(0.972)$ \\
\hline \multirow[t]{2}{*}{ Share of fruits } & & & & & $-4.386^{\star *}$ & $-4.671^{* * *}$ & $-3.672^{*}$ & $-3.891^{* * *}$ \\
\hline & & & & & $(2.235)$ & $(1.105)$ & (2.159) & $(1.120)$ \\
\hline \multirow{2}{*}{$\begin{array}{l}\text { Share of roots and } \\
\text { tubers }\end{array}$} & & & & & -1.420 & -0.757 & -0.772 & -0.0388 \\
\hline & & & & & (1.611) & $(1.656)$ & (1.402) & (1.589) \\
\hline \multirow[t]{2}{*}{ Share of coarse grain } & & & & & $-2.609^{* *}$ & $-2.311^{* * *}$ & $-2.136^{\star}$ & $-1.902^{\star * *}$ \\
\hline & & & & & $(1.316)$ & $(0.741)$ & (1.235) & $(0.722)$ \\
\hline \multirow[t]{2}{*}{ Share of oil crops } & & & & & -1.858 & -1.169 & -1.359 & -0.569 \\
\hline & & & & & (1.352) & $(0.857)$ & $(1.254)$ & $(0.810)$ \\
\hline Constant & $\begin{array}{l}-12.90^{\star * \star} \\
(4.810)\end{array}$ & $\begin{array}{l}-13.92^{\star \star \star} \\
(1.855)\end{array}$ & $\begin{array}{l}-12.49^{\star *} \\
(5.017)\end{array}$ & $\begin{array}{l}-13.99^{\star \star *} \\
(1.906)\end{array}$ & $\begin{array}{l}-10.95^{\star *} \\
(4.900)\end{array}$ & $\begin{array}{l}-11.71^{\star * \star} \\
(2.208)\end{array}$ & $\begin{array}{l}-11.31^{* *} \\
(5.065)\end{array}$ & $\begin{array}{l}-12.64^{\star \star \star} \\
(2.170)\end{array}$ \\
\hline $\mathrm{N}$ & 1376 & 1376 & 1376 & 1376 & 1376 & 1376 & 1376 & 1376 \\
\hline R-sq & & 0.923 & & 0.927 & & 0.926 & & 0.930 \\
\hline
\end{tabular}

Note: Robust standard errors appear in parentheses; asterisks *, **, and *** indicate significance at the $10 \%, 5 \%$, and $1 \%$ levels, respectively.

Table 4 - Estimation results for pesticide use intensity

\begin{tabular}{|c|c|c|c|c|c|c|c|c|}
\hline & $\mathrm{RE}$ & PCSE & $\mathrm{RE}$ & PCSE & $\mathrm{RE}$ & PCSE & $\mathrm{RE}$ & PCSE \\
\hline \multirow[t]{2}{*}{ InGDP } & $2.405^{*}$ & $2.137^{* * *}$ & $2.627^{* *}$ & $2.598^{* * *}$ & $2.450^{* *}$ & $2.322^{* * *}$ & $2.545^{\star *}$ & $2.583^{* * *}$ \\
\hline & $(1.241)$ & $(0.210)$ & $(1.272)$ & $(0.215)$ & $(1.168)$ & $(0.221)$ & $(1.200)$ & $(0.220)$ \\
\hline \multirow[t]{2}{*}{$(\operatorname{lnGDP})^{2}$} & -0.101 & $-0.0826^{\star * *}$ & $-0.128^{*}$ & $-0.134^{* * *}$ & $-0.110^{*}$ & $-0.102^{\star * *}$ & $-0.123^{*}$ & $-0.133^{\star * *}$ \\
\hline & (0.0707) & $(0.0123)$ & $(0.0748)$ & $(0.0131)$ & $(0.0660)$ & $(0.0136)$ & (0.0702) & $(0.0135)$ \\
\hline Country's fixed effect & Yes & Yes & Yes & Yes & Yes & Yes & Yes & Yes \\
\hline Year fixed effect & No & No & Yes & Yes & No & No & Yes & Yes \\
\hline Share of cereals & & & & & $\begin{array}{l}-1.861 \\
(1.873)\end{array}$ & $\begin{array}{l}-1.803^{\text {*** }} \\
(0.641)\end{array}$ & $\begin{array}{l}-1.880 \\
(1.886)\end{array}$ & $\begin{array}{l}-1.951^{* * *} \\
(0.641)\end{array}$ \\
\hline \multirow[t]{2}{*}{ Share of vegetables } & & & & & 0.466 & 0.725 & 0.145 & 0.0420 \\
\hline & & & & & $(2.316)$ & $(0.968)$ & (2.196) & $(0.952)$ \\
\hline \multirow[t]{2}{*}{ Share of fruits } & & & & & 1.013 & $1.373^{*}$ & 0.956 & 1.115 \\
\hline & & & & & $(2.418)$ & $(0.779)$ & $(2.411)$ & $(0.772)$ \\
\hline \multirow{2}{*}{$\begin{array}{l}\text { Share of roots and } \\
\text { tubers }\end{array}$} & & & & & 0.360 & 0.995 & 0.200 & 0.375 \\
\hline & & & & & (2.272) & $(0.787)$ & (2.119) & $(0.767)$ \\
\hline \multirow[t]{2}{*}{ Share of coarse grain } & & & & & -1.944 & $-1.744^{* *}$ & -1.829 & $-1.867^{\star * *}$ \\
\hline & & & & & (1.918) & $(0.694)$ & (1.923) & $(0.706)$ \\
\hline \multirow[t]{2}{*}{ Share of oil crops } & & & & & 0.905 & $1.279^{*}$ & 0.622 & 0.787 \\
\hline & & & & & $(1.770)$ & $(0.660)$ & $(1.726)$ & $(0.661)$ \\
\hline Constant & $\begin{array}{l}-12.75^{\star *} \\
(5.366)\end{array}$ & $\begin{array}{l}-13.13^{* * *} \\
(0.903)\end{array}$ & $\begin{array}{l}-12.68^{* *} \\
(5.357)\end{array}$ & $\begin{array}{l}-13.84^{* * *} \\
(0.919)\end{array}$ & $\begin{array}{l}-11.44^{* *} \\
(5.376)\end{array}$ & $-12.48^{\star * *}$ & $\begin{array}{l}-11.27^{\star *} \\
(5.386)\end{array}$ & $-12.58^{\star \star *}$ \\
\hline $\mathrm{N}$ & 2408 & 2408 & 2408 & 2408 & 2408 & 2408 & 2408 & 2408 \\
\hline R-sq & & 0.886 & & 0.888 & & 0.891 & & 0.893 \\
\hline
\end{tabular}

Note: Robust standard errors appear in parentheses; asterisks *, ${ }^{* *}$, and ${ }^{* *}$ indicate significance at the $10 \%, 5 \%$, and $1 \%$ levels, respectively. 
Table 5 - Estimation results for cropping frequency

\begin{tabular}{|c|c|c|c|c|c|c|c|c|}
\hline & $\mathrm{RE}$ & PCSE & $\mathrm{RE}$ & PCSE & $\mathrm{RE}$ & PCSE & RE & PCSE \\
\hline InGDP & $\begin{array}{l}0.349^{*} \\
(0.204)\end{array}$ & $\begin{array}{l}0.374^{* * *} \\
(0.0523)\end{array}$ & $\begin{array}{l}0.363^{*} \\
(0.201)\end{array}$ & $\begin{array}{l}0.375^{* * *} \\
(0.0563)\end{array}$ & $\begin{array}{l}0.395^{*} \\
(0.215)\end{array}$ & $0.416^{* * *}$ & $\begin{array}{l}0.433^{* *} \\
(0.208)\end{array}$ & $0.455^{\star * *}$ \\
\hline$(\operatorname{lnGDP})^{2}$ & $\begin{array}{l}-0.0162 \\
(0.0125)\end{array}$ & $\begin{array}{l}-0.0171^{\star * *} \\
(0.00339)\end{array}$ & $\begin{array}{l}-0.0182 \\
(0.0123)\end{array}$ & $\begin{array}{l}-0.0175^{\star * *} \\
(0.00389)\end{array}$ & $\begin{array}{l}-0.0180 \\
(0.0132)\end{array}$ & $\begin{array}{l}-0.0183^{\star * *} \\
(0.00379)\end{array}$ & $\begin{array}{l}-0.0233^{*} \\
(0.0125)\end{array}$ & $\begin{array}{l}-0.0233^{* * *} \\
(0.00451)\end{array}$ \\
\hline $\begin{array}{l}\text { Country's fixed } \\
\text { effect }\end{array}$ & Yes & Yes & Yes & Yes & Yes & Yes & Yes & Yes \\
\hline $\begin{array}{l}\text { Year fixed effect } \\
\text { Share of cereals }\end{array}$ & No & No & Yes & Yes & $\begin{array}{l}\text { No } \\
0.218 \\
(0.364)\end{array}$ & $\begin{array}{l}\text { No } \\
0.292^{\star * *} \\
(0.113)\end{array}$ & $\begin{array}{l}\text { Yes } \\
0.177 \\
(0.364)\end{array}$ & $\begin{array}{l}\text { Yes } \\
0.243^{* *} \\
(0.118)\end{array}$ \\
\hline $\begin{array}{l}\text { Share of } \\
\text { vegetables }\end{array}$ & & & & & $\begin{array}{l}-1.238^{* *} \\
(0.570)\end{array}$ & $\begin{array}{l}-1.178^{\star \star \star} \\
(0.284)\end{array}$ & $\begin{array}{l}-1.399^{* *} \\
(0.575)\end{array}$ & $\begin{array}{l}-1.320^{* * *} \\
(0.287)\end{array}$ \\
\hline Share of fruits & & & & & $\begin{array}{l}-0.699 \\
(0.615)\end{array}$ & $\begin{array}{l}-0.676^{\star \star \star} \\
(0.200)\end{array}$ & $\begin{array}{l}-0.759 \\
(0.642)\end{array}$ & $\begin{array}{l}-0.739^{* * *} \\
(0.199)\end{array}$ \\
\hline $\begin{array}{l}\text { Share of roots and } \\
\text { tubers }\end{array}$ & & & & & $\begin{array}{l}-1.031^{*} \\
(0.598)\end{array}$ & $\begin{array}{l}-0.958^{\star \star \star} \\
(0.315)\end{array}$ & $\begin{array}{l}-1.180^{\star *} \\
(0.569)\end{array}$ & $\begin{array}{l}-1.108^{\star \star \star} \\
(0.310)\end{array}$ \\
\hline $\begin{array}{l}\text { Share of coarse } \\
\text { grain }\end{array}$ & & & & & $\begin{array}{l}0.945^{\star \star} \\
(0.465)\end{array}$ & $\begin{array}{l}1.053^{\star * \star} \\
(0.126)\end{array}$ & $\begin{array}{l}0.917^{\star \star} \\
(0.467)\end{array}$ & $\begin{array}{l}1.003^{* * *} \\
(0.126)\end{array}$ \\
\hline Share of oil crops & & & & & $\begin{array}{l}0.309 \\
(0.415)\end{array}$ & $\begin{array}{l}0.369^{\star \star \star} \\
(0.122)\end{array}$ & $\begin{array}{l}0.175 \\
(0.439)\end{array}$ & $\begin{array}{l}0.253^{\star *} \\
(0.125)\end{array}$ \\
\hline Constant & $\begin{array}{l}-1.953^{\star *} \\
(0.829)\end{array}$ & $\begin{array}{l}-2.177^{\star * *} \\
(0.205)\end{array}$ & $\begin{array}{l}-1.927^{\star *} \\
(0.836)\end{array}$ & $\begin{array}{l}-2.159^{\star \star *} \\
(0.212)\end{array}$ & $\begin{array}{l}-2.396^{* *} \\
(0.974)\end{array}$ & $\begin{array}{l}-2.535^{\star \star *} \\
(0.257)\end{array}$ & $\begin{array}{l}-2.286^{* *} \\
(0.968)\end{array}$ & $\begin{array}{l}-2.496^{* * *} \\
(0.270)\end{array}$ \\
\hline $\mathrm{N}$ & 2408 & 2408 & 2408 & 2408 & 2408 & 2408 & 2408 & 2408 \\
\hline$R$-sq & & 0.934 & & 0.935 & & 0.942 & & 0.943 \\
\hline
\end{tabular}

Note: Robust standard errors appear in parentheses; asterisks *, ${ }^{* *}$, and ${ }^{* * *}$ indicate significance at the $10 \%, 5 \%$, and $1 \%$ levels, respectively.

Table 6 - Estimation results for production value of crops per area

\begin{tabular}{|c|c|c|c|c|c|c|c|c|}
\hline & RE & PCSE & $\mathrm{RE}$ & PCSE & RE & PCSE & RE & PCSE \\
\hline InGDP & $1.044^{\star \star \star}$ & $1.011^{* * *}$ & $\begin{array}{l}1.355^{\star * *} \\
(0350)\end{array}$ & $1.353^{* * *}$ & $\begin{array}{l}1.254^{* * *} \\
(0326)\end{array}$ & $1.224^{* * *}$ & $\begin{array}{l}1.458^{* * \star} \\
(0319)\end{array}$ & $1.475^{\star * *}$ \\
\hline$(\operatorname{lnGDP})^{2}$ & $\begin{array}{l}-0.0320 \\
(0.0208)\end{array}$ & $\begin{array}{l}-0.0283^{* * *} \\
(0.00595)\end{array}$ & $\begin{array}{l}-0.0677^{\star \star \star} \\
(0.0215)\end{array}$ & $\begin{array}{l}-0.0666^{\star * *} \\
(0.00619)\end{array}$ & $\begin{array}{l}-0.0490^{\star *} \\
(0.0197)\end{array}$ & $\begin{array}{l}-0.0445^{\star \star \star} \\
(0.00579)\end{array}$ & $\begin{array}{l}-0.0752^{\star * *} \\
(0.0196)\end{array}$ & $\begin{array}{l}-0.0749^{\star \star \star} \\
(0.00626)\end{array}$ \\
\hline $\begin{array}{l}\text { Country's } \\
\text { fixed effect }\end{array}$ & Yes & Yes & Yes & Yes & Yes & Yes & Yes & Yes \\
\hline $\begin{array}{l}\text { Year fixed } \\
\text { effect }\end{array}$ & No & No & Yes & Yes & No & No & Yes & Yes \\
\hline $\begin{array}{l}\text { Share of } \\
\text { cereals }\end{array}$ & & & & & $\begin{array}{l}0.511 \\
(0.451)\end{array}$ & $\begin{array}{l}0.616^{\star \star *} \\
(0.182)\end{array}$ & $\begin{array}{l}0.440 \\
(0.413)\end{array}$ & $\begin{array}{l}0.492^{\star \star *} \\
(0.185)\end{array}$ \\
\hline $\begin{array}{l}\text { Share of } \\
\text { vegetables }\end{array}$ & & & & & $\begin{array}{l}1.725^{\star * *} \\
(0.527)\end{array}$ & $\begin{array}{l}1.709^{* * *} \\
(0.302)\end{array}$ & $\begin{array}{l}1.012^{* *} \\
(0.441)\end{array}$ & $\begin{array}{l}0.935^{\star * *} \\
(0.247)\end{array}$ \\
\hline Share of fruits & & & & & $\begin{array}{l}1.497^{* *} \\
(0.735)\end{array}$ & $\begin{array}{l}1.544^{\star * *} \\
(0.265)\end{array}$ & $\begin{array}{l}1.304^{*} \\
(0.784)\end{array}$ & $\begin{array}{l}1.286^{\star \star *} \\
(0.256)\end{array}$ \\
\hline $\begin{array}{l}\text { Share of roots } \\
\text { and tubers }\end{array}$ & & & & & $\begin{array}{l}0.865 \\
(0.695)\end{array}$ & $\begin{array}{l}1.075^{\star * *} \\
(0.240)\end{array}$ & $\begin{array}{l}0.348 \\
(0.640)\end{array}$ & $\begin{array}{l}0.420 \\
(0.262)\end{array}$ \\
\hline $\begin{array}{l}\text { Share of } \\
\text { coarse grain }\end{array}$ & & & & & $\begin{array}{l}-0.600 \\
(0.578)\end{array}$ & $\begin{array}{l}-0.290 \\
(0.226)\end{array}$ & $\begin{array}{l}-0.540 \\
(0.558)\end{array}$ & $\begin{array}{l}-0.365^{*} \\
(0.206)\end{array}$ \\
\hline $\begin{array}{l}\text { Share of oil } \\
\text { crops }\end{array}$ & & & & & $\begin{array}{l}0.927^{\star *} \\
(0.448)\end{array}$ & $\begin{array}{l}1.041^{* * *} \\
(0.181)\end{array}$ & $\begin{array}{l}0.418 \\
(0.443)\end{array}$ & $\begin{array}{l}0.509^{* * *} \\
(0.177)\end{array}$ \\
\hline Constant & $\begin{array}{l}0.123 \\
(1.469)\end{array}$ & $\begin{array}{l}0.978^{\star \star *} \\
(0.352)\end{array}$ & $\begin{array}{l}0.0423 \\
(1.435)\end{array}$ & $\begin{array}{l}0.457 \\
(0.332)\end{array}$ & $\begin{array}{l}-0.824 \\
(1.426)\end{array}$ & $\begin{array}{l}-0.355 \\
(0.390)\end{array}$ & $\begin{array}{l}-0.549 \\
(1.352)\end{array}$ & $\begin{array}{l}-0.415 \\
(0.407)\end{array}$ \\
\hline $\begin{array}{l}\mathrm{N} \\
\mathrm{R} \text {-sq }\end{array}$ & 2322 & $\begin{array}{l}2322 \\
0.953\end{array}$ & 2322 & $\begin{array}{l}2322 \\
0.959\end{array}$ & 2322 & $\begin{array}{l}2322 \\
0.955\end{array}$ & 2322 & $\begin{array}{l}2322 \\
0.960\end{array}$ \\
\hline
\end{tabular}

Note: Robust standard errors appear in parentheses; asterisks * **, and ${ }^{* * *}$ indicate significance at the $10 \%, 5 \%$, and $1 \%$ levels, respectively.

The estimated coefficients are positive for the linear term and negative for squared term, which suggests the existence of an inverted-U-shaped relationship between per capita GDP and fertilizer use intensity. This is consistent with the EKC hypothesis. The estimated turning point is 24407.154 US $\$$ (2015 prices). This means that as per capita income increases at the initial stages, fertilizer use intensity follows the same path but begins to decline after reaching the turning point.

The estimation results for pesticide use intensity are provided in Table 4. The estimated coefficients are positive for the linear term and negative for squared term, which suggests the existence of an inverted-U-shaped relationship between per capita GDP and pesticide use intensity. The estimated turning point is 16490.279 US\$ (2015 prices). This means that in the 
early stages of economic development, pesticide use intensity rises steadily as per capita income grows, and then begins to decrease as per capita income continues to grow once the turning point is reached.

The estimation results for cropping frequency are shown in Table 5. The results suggest the existence of an inverted-U-shaped relationship between per capita GDP and cropping frequency and the validity of the EKC. The estimated turning point is 17395.181 US\$ (2015 prices). In other words, as per capita income increases, cropping frequency also increases at first and then starts declining after the turning point.

The estimation results for production value of crops per area are presented in Table 6. The results indicate that there is an inverted-U-shaped relationship between per capita GDP and production value of crops per area, and confirm the EKC hypothesis. The estimated turning point is 18891.398 US\$ (2015 prices).

The estimation results for GHG emissions per area are provided in Table 7 . The results confirm the EKC hypothesis. The estimated turning point is 17387.489 US $\$$ (2015 prices). This means that GHG emissions per area increases faster than per capita GDP in the early stages of economic growth and slows down relative to per capita GDP growth in higher per capita income levels.

Table 7 - Estimation results for GHG emissions per area

\begin{tabular}{|c|c|c|c|c|c|c|c|c|}
\hline & $\mathrm{RE}$ & PCSE & $\mathrm{RE}$ & PCSE & $\mathrm{RE}$ & PCSE & $\mathrm{RE}$ & PCSE \\
\hline \multirow[t]{2}{*}{ InGDP } & $0.779^{\star \star}$ & $0.797^{\star \star \star}$ & $0.874^{* * *}$ & $0.915^{\star \star \star}$ & $0.747^{\star \star}$ & $0.767^{\star \star \star}$ & $0.846^{\star * *}$ & $0.900^{\star * *}$ \\
\hline & $(0.339)$ & $(0.0516)$ & $(0.337)$ & $(0.0527)$ & $(0.322)$ & $(0.0514)$ & $(0.319)$ & $(0.0580)$ \\
\hline \multirow[t]{2}{*}{$(\operatorname{lnGDP})^{2}$} & -0.0319 & $-0.0327^{* * *}$ & $-0.0436^{* *}$ & $-0.0466^{* * *}$ & -0.0295 & $-0.0302^{\star \star \star *}$ & $-0.0419^{* *}$ & $-0.0460^{* * *}$ \\
\hline & $(0.0202)$ & $(0.00312)$ & $(0.0204)$ & $(0.00340)$ & $(0.0195)$ & $(0.00313)$ & $(0.0196)$ & $(0.00388)$ \\
\hline Country's fixed effect & Yes & Yes & Yes & Yes & Yes & Yes & Yes & Yes \\
\hline Year fixed effect & No & No & Yes & Yes & No & No & Yes & Yes \\
\hline Share of cereals & & & & & $\begin{array}{l}0.301 \\
(0.518)\end{array}$ & $\begin{array}{l}0.349^{\star * *} \\
(0.135)\end{array}$ & $\begin{array}{l}0.359 \\
(0.512)\end{array}$ & $\begin{array}{l}0.388^{* * *} \\
(0.137)\end{array}$ \\
\hline \multirow[t]{2}{*}{ Share of vegetables } & & & & & 0.687 & $0.639^{* * *}$ & 0.469 & $0.367^{* *}$ \\
\hline & & & & & $(0.708)$ & $(0.197)$ & $(0.659)$ & $(0.183)$ \\
\hline \multirow[t]{2}{*}{ Share of fruits } & & & & & 0.425 & $0.427^{\star *}$ & 0.440 & $0.413^{* *}$ \\
\hline & & & & & $(0.608)$ & $(0.194)$ & $(0.609)$ & $(0.191)$ \\
\hline \multirow[t]{2}{*}{ Share of roots and tubers } & & & & & 0.500 & $0.467^{* *}$ & 0.280 & 0.166 \\
\hline & & & & & $(0.647)$ & $(0.238)$ & $(0.601)$ & $(0.246)$ \\
\hline \multirow[t]{2}{*}{ Share of coarse grain } & & & & & 0.00615 & 0.0846 & -0.00530 & 0.0184 \\
\hline & & & & & $(0.559)$ & $(0.177)$ & $(0.553)$ & $(0.186)$ \\
\hline \multirow[t]{2}{*}{ Share of oil crops } & & & & & -0.132 & -0.107 & -0.297 & $-0.302^{\star *}$ \\
\hline & & & & & $(0.482)$ & $(0.128)$ & $(0.471)$ & $(0.133)$ \\
\hline \multirow[t]{2}{*}{ Constant } & $2.910^{* *}$ & $3.036^{\star * *}$ & $2.975^{\star *}$ & $2.931^{* * *}$ & $2.801^{* *}$ & $2.794^{* * *}$ & $2.892^{* *}$ & $2.708^{* * *}$ \\
\hline & $(1.401)$ & $(0.223)$ & $(1.382)$ & $(0.233)$ & $(1.385)$ & $(0.247)$ & $(1.356)$ & $(0.292)$ \\
\hline $\mathrm{N}$ & 2408 & 2408 & 2408 & 2408 & 2408 & 2408 & 2408 & 2408 \\
\hline R-sq & & 0.950 & & 0.952 & & 0.951 & & 0.953 \\
\hline
\end{tabular}

Note: Robust standard errors appear in parentheses; asterisks *, **, and *** indicate significance at the $10 \%$, 5\%, and $1 \%$ levels, respectively.

\section{DISCUSSION OF RESULTS}

To better understand the impact of economic growth on agricultural land-use intensity, we continue to discuss the potential causes behind the EKC for agricultural land-use intensity. According to (Dinda, 2004), several factors are responsible to shape the EKC, including income elasticity of environmental quality demand, international trade, market mechanism, regulation, etc. For the agricultural land-use intensity indicators, fertilizer use intensity, pesticide use intensity, and GHG emissions per area are closely related to environmental degradation, thus, as income grows, people achieve a higher standard of living and care more for the quality of environment they live in and demand for better environment induces structural changes in agriculture that tends to reduce agricultural chemical use and GHG emissions. Increasing food demand requires more input including farmland, fertilizer, pesticide, labor, energy, etc. Therefore, agricultural land-use intensity will be affected by population and income level that affect food demand. On the other hand, technological progress occurs with economic growth and the obsolete and dirty technologies are replaced by upgraded new and cleaner technology, which improves environmental quality. This is the 
technique effect of economic growth (Dinda, 2004). Sustainable intensification has been gaining attention in policy discussions as an appropriate means to use land in order to increase food supplies while protecting environmental security (Petersen and Snapp, 2015). Meanwhile, because the adoption of technological innovations and modern management practices involves high fixed costs, the adoption rate of small-scale farms is relatively low (Feder et al., 1985). Some empirical studies highlighted the importance of farm size in understanding changes in land use intensity (van der Sluis et al., 2016; Wu et al., 2018; Zhu and Wang, 2020). Because land resources are limited, the expansion of farm size is mainly due to the transfer of agricultural labor to other industries. (Liu et al., 2016) found there is an inverted-N-shaped relationship between rural out-migration and arable land use intensity. While (Gray and Bilsborrow, 2014) found that migrant departure has a positive effect on agricultural activities. (Caulfield et al., 2019) demonstrated that remittances received from rural out-migrations were associated with an increase in the use of pesticides and chemical fertilizers. (Zhang et al., 2020) found that off-farm employment has a significant influence on chemical fertilizer use. (Jiang et al., 2013) revealed that urban expansion driven by population urbanization decreased the agricultural land use intensity. While (You et al., 2018) found that urban population proportion has a positive effect on input intensity.

Table 8 - Estimation results for mediation analysis

\begin{tabular}{|c|c|c|c|c|c|c|c|c|}
\hline & Total pop. & $\begin{array}{l}\text { Urban } \\
\text { pop. }\end{array}$ & $\begin{array}{l}\text { Farm } \\
\text { size }\end{array}$ & Fertilizer & Pesticide & Currency & Value & Emission \\
\hline InGDP & $\begin{array}{l}0.680^{* * *} \\
(0.0385)\end{array}$ & $\begin{array}{l}0.570^{* * *} \\
(0.0244)\end{array}$ & $\begin{array}{l}-0.264^{\star * *} \\
(0.0533)\end{array}$ & $\begin{array}{l}2.523^{\star * *} \\
(0.430)\end{array}$ & $\begin{array}{l}2.668^{\star * *} \\
(0265)\end{array}$ & $\begin{array}{l}0.410^{\star * \star} \\
(0.0661)\end{array}$ & $\begin{array}{l}1.037^{* * *} \\
(0.0844)\end{array}$ & $\begin{array}{l}0.515^{\star * *} \\
(0.0628)\end{array}$ \\
\hline$(\operatorname{lnGDP})^{2}$ & $\begin{array}{l}-0.0556^{\star \star \star} \\
(0.00356)\end{array}$ & $\begin{array}{l}-0.0360^{* * *} \\
(0.00152)\end{array}$ & $\begin{array}{l}0.0249^{\star * *} \\
(0.00393)\end{array}$ & $\begin{array}{l}-0.0988^{* * *} \\
(0.0252)\end{array}$ & $\begin{array}{l}-0.133^{\star * *} \\
(0.0162)\end{array}$ & $\begin{array}{l}-0.0187^{\star \star \star} \\
(0.00450)\end{array}$ & $\begin{array}{l}-0.0460^{\star \star \star} \\
(0.00582)\end{array}$ & $\begin{array}{l}-0.0149^{\star \star \star} \\
(0.00433)\end{array}$ \\
\hline In(total pop.) & & & & $\begin{array}{l}4.702^{\star * \star} \\
(1.180)\end{array}$ & $\begin{array}{l}2.571^{* * *} \\
(0.422)\end{array}$ & $\begin{array}{l}0.0820 \\
(0.111)\end{array}$ & $\begin{array}{l}-1.125^{\star \star \star} \\
(0.108)\end{array}$ & $\begin{array}{l}0.240 \\
(0.151)\end{array}$ \\
\hline$(\ln (\text { total pop. }))^{2}$ & & & & $\begin{array}{l}-0.235^{\star * *} \\
(0.0694)\end{array}$ & $\begin{array}{l}-0.147^{* * *} \\
(0.0219)\end{array}$ & $\begin{array}{l}-0.00925^{*} \\
(0.00558)\end{array}$ & $\begin{array}{l}0.0673^{\star * *} \\
(0.00508)\end{array}$ & $\begin{array}{l}0.000776 \\
(0.00875)\end{array}$ \\
\hline In(urban pop.) & & & & $\begin{array}{l}0.730 \\
(0.456)\end{array}$ & 0.0929 & $0.317^{\star * *}$ & $\begin{array}{l}1.153^{* * *} \\
(0101)\end{array}$ & $1.031^{* * *}$ \\
\hline$\left(\ln (\text { urban pop.) })^{2}\right.$ & & & & $\begin{array}{l}-0.504^{* * *} \\
(0.0777)\end{array}$ & $\begin{array}{l}-0.180^{* *} \\
(0.0761)\end{array}$ & $\begin{array}{l}0.0544^{* \star *} \\
(0.0195)\end{array}$ & $\begin{array}{l}0.244^{* * *} \\
(0.0285)\end{array}$ & $\begin{array}{l}0.210^{* * *} \\
(0.0264)\end{array}$ \\
\hline In(farm size) & & & & $\begin{array}{l}-0.646^{* * *} \\
(0.119)\end{array}$ & $\begin{array}{l}0.0685 \\
(0.0511)\end{array}$ & $\begin{array}{l}-0.122^{* * *} \\
(0.0158)\end{array}$ & $\begin{array}{l}-0.262^{* \star *} \\
(0.0224)\end{array}$ & $\begin{array}{l}-0.550^{\star \star *} \\
(0.0146)\end{array}$ \\
\hline$(\ln (\text { farm size }))^{2}$ & & & & $\begin{array}{l}0.0919^{\star \star \star} \\
(0.0333)\end{array}$ & $\begin{array}{l}0.114^{\star * \star} \\
(0.0277)\end{array}$ & $\begin{array}{l}0.0690^{\star \star \star *} \\
(0.00532)\end{array}$ & $\begin{array}{l}0.0347^{\star \star \star} \\
(0.00678)\end{array}$ & $\begin{array}{l}-0.00307 \\
(0.00786)\end{array}$ \\
\hline $\begin{array}{l}\text { Control of categories } \\
\text { of crops }\end{array}$ & No & No & No & Yes & Yes & Yes & Yes & Yes \\
\hline Country's fixed effect & Yes & Yes & Yes & Yes & Yes & Yes & Yes & Yes \\
\hline Year fixed effect & Yes & Yes & Yes & Yes & Yes & Yes & Yes & Yes \\
\hline Constant & $\begin{array}{l}5.856^{\star \star *} \\
(0.118)\end{array}$ & $\begin{array}{l}-2.774^{\star * *} \\
(0.107)\end{array}$ & $\begin{array}{l}-0.131 \\
(0.196)\end{array}$ & $\begin{array}{l}-30.91^{\star * *} \\
(6.382)\end{array}$ & $\begin{array}{l}-24.49^{* * *} \\
(2.575)\end{array}$ & $\begin{array}{l}-2.437^{\star * *} \\
(0.659)\end{array}$ & $\begin{array}{l}6.675^{\star \star *} \\
(0.791)\end{array}$ & $\begin{array}{l}2.119^{* *} \\
(0.930)\end{array}$ \\
\hline $\mathrm{N}$ & 2408 & 2408 & 2408 & 1376 & 2408 & 2408 & 2322 & 2408 \\
\hline R-sq & 0.998 & 0.991 & 0.980 & 0.938 & 0.895 & 0.947 & 0.965 & 0.968 \\
\hline
\end{tabular}

Note: Robust standard errors appear in parentheses; asterisks ${ }^{*},{ }^{* *}$, and ${ }^{* *}$ indicate significance at the $10 \%, 5 \%$, and $1 \%$ levels, respectively.

The literature shows that various economic indicators, such as total population, urban population proportion, farm size, maybe potential mediators of the effects of economic growth. In order to test the effect of these potential mediators, we perform a mediation analysis (Mackinnon and Fairchild, 2009). Due to the availability of data, we detect the mediated effects of total population, urban population proportion, and farm size. The cropland area per rural population is used as the proxy variable of farm size. The data for our analysis were extracted from FAO database (FAOSTAT, 2017). The brief estimation results for mediation analysis are shown in Table 8, and the detailed estimation results are provided in Tables S1-S6 in Appendix. According to (Preacher and Hayes, 2008), the estimation results of separate models and one model including all mediators indicate that total population, urban population proportion, and farm size are mediators of the effects of economic growth on the agricultural land-use intensity. 


\section{CONCLUSION}

This study investigates the relationship between economic growth and agricultural land-use intensity indicators, including fertilizer use intensity, pesticide use intensity, cropping frequency, production value of crops per area, and GHG emissions per area, using data of 86 countries in 1990-2017. The results demonstrate the existence of an inverted-U-shaped relationship between economic growth and each of the agricultural land-use intensity indicators, which is consistent with the EKC hypothesis. This implies that in the early stages of economic growth, agricultural land-use intensity rises steadily as per capita income grows, and then begins to decline as per capita income continues to grow after reaching the turning point. Furthermore, the mediation analysis shows that total population, urban population proportion, and farm size are mediators of the effects of economic growth on the agricultural land-use intensity.

It should be noted that this study has several limitations. First, the indicators of agricultural land-use intensity other than the five indicators analyzed above have not been discussed in this study. Second, some of the variables, such as cropping frequency and farm size, are proxy variables, so that there may be measurement errors, that may lead to inaccurate estimates of these variables. Third, the potential mediated effects of technological progress, government expenditure on agriculture, foreign direct investment, etc. have not been tested in this study. In future research, more accurate and detailed data can be used to address the above limitations.

\section{ACKNOWLEDGEMENTS}

This work was supported by the National Social Science Fund of China [16AJY013].

\section{REFERENCES}

1. Ali G, Ashraf A, Bashir MK, Cui S. Exploring environmental Kuznets curve in relation to green revolution: A case study of Pakistan. Env. Science \& Policy 2017; 77: 166-171.

2. Bimonte S, Stabile A. Land consumption and income in Italy: a case of inverted EKC. Ecological Economics 2017; 131: 36-43.

3. Cassou E. Pesticides. Agricultural Pollution. The Challenge of Agricultural Pollution: Evidence from China, Vietnam, and the Philippines. World Bank, Washington, DC., 2018.

4. Caulfield M, Bouniol J, Fonte SJ, Kessler A. How rural out-migrations drive changes to farm and land management: A case study from the rural Andes. Land Use Policy 2019; 81: 594-603.

5. Dinda S. Environmental Kuznets Curve Hypothesis: A Survey. Ecological Economics 2004; 49: 431-455.

6. EPA. Report on the Environment. https://www.epa.gov/report-environment/ land-use\#note4 [accessed 10 July 2020], 2020.

7. Erb KH, Haberl H, Jepsen MR, Kuemmerle T, Lindner M, Muller D, et al. A conceptual framework for analysing and measuring land-use intensity. Current Opinion in Environmental Sustainability 2013; 5: 464-470.

8. FAO, IFAD, UNICEF, WFP, WHO. The State of Food Security and Nutrition in the World 2019. FAO, Rome, 2019.

9. FAO Statistical Databases. http://www.fao.org/faostat/en/ (accessed 17 March 2020).

10. Feder G, Just RE, Zilberman D. Adoption of agricultural innovations in developing countries: A survey. Economic development and cultural change 1985; 33: 255-298.

11. Ghimire N, Woodward RT. Under- and over-use of pesticides: An international analysis. Ecological Economics 2013; 89: 73-81.

12. Godfray HC, Beddington JR, Crute IR, Haddad L, Lawrence D, Muir JF, et al. Food security: the challenge of feeding 9 billion people. Science 2010; 327: 812-8.

13. Gray CL, Bilsborrow RE. Consequences of Out-Migration for Land Use in Rural Ecuador. Land use policy $2014 ; 36$.

14. Grossman GM, Krueger AB. Economic growth and the environment. Quarterly Journal of 
Economics 1995; 110: 353-377.

15. Hedlund J, Longo SB, York R. Agriculture, Pesticide Use, and Economic Development: A Global Examination (1990-2014) Rural Sociology 2020; 85: 519-544.

16. Jiang $L$, Deng $X$, Seto KC. The impact of urban expansion on agricultural land use intensity in China. Land Use Policy 2013; 35: 33-39.

17. Kuznets S. Economic growth and income inequality. Amer. Economic Review 1955: 1-28.

18. Lambin EF, Gibbs HK, Ferreira L, Grau R, Mayaux P, Meyfroidt P, et al. Estimating the world's potentially available cropland using a bottom-up approach. Global Environmental Change 2013; 23: 892-901.

19. Lambin EF, Meyfroidt P. Global land use change, economic globalization, and the looming land scarcity. Proc Natl Acad Sci USA 2011; 108: 3465-72.

20. Liu G, Wang H, Cheng Y, Zheng B, Lu Z. The impact of rural out-migration on arable land use intensity: Evidence from mountain areas in Guangdong, China. Land Use Policy 2016; 59: 569-579.

21. Mackinnon DP, Fairchild AJ. Current Directions in Mediation Analysis. Curr Dir Psychol Sci 2009; 18: 16.

22. Managi S. Are there increasing returns to pollution abatement? Empirical analytics of the Environmental Kuznets Curve in pesticides. Ecological Economics 2006; 58: 617-636.

23. Pellegrini P, Fernandez RJ. Crop intensification, land use, and on-farm energy-use efficiency during the worldwide spread of the green revolution. Proc Natl Acad Sci U S A 2018; 115: 2335-2340.

24. Petersen B, Snapp S. What is sustainable intensification? Views from experts. Land Use Policy 2015; 46: 1-10.

25. Pontarollo N, Serpieri C. Testing the Environmental Kuznets Curve hypothesis on land use: The case of Romania. Land Use Policy 2020; 97.

26. Preacher KJ, Hayes AF. Asymptotic and resampling strategies for assessing and comparing indirect effects in multiple mediator models. Behav Res Methods 2008; 40: 879-91.

27. Reay DS, Davidson EA, Smith KA, Smith P, Melillo JM, Dentener F, et al. Global agriculture and nitrous oxide emissions. Nature Climate Change 2012; 2: 410-416.

28. RodríGuez-Meza J, Southgate D, GonzÁLez-Vega C. Rural poverty, household responses to shocks, and agricultural land use: panel results for El Salvador. Environment and Development Economics 2004; 9: 225-239.

29. Sarkodie SA, Strezov V. A review on Environmental Kuznets Curve hypothesis using bibliometric and meta-analysis. Science of the Total Environment 2019; 649: 128-145.

30. Schreinemachers P, Tipraqsa P. Agricultural pesticides and land use intensification in high, middle and low income countries. Food Policy 2012; 37: 616-626.

31. Singh AP, Narayanan K. Impact of economic growth and population on agrochemical use: evidence from post-liberalization India. Environment, Development and Sustainability 2015; 17: 1509-1525.

32. Stern DI. The Rise and Fall of the Environmental Kuznets Curve. World Development 2004; 32: 1419-1439.

33. Tilman D, Balzer C, Hill J, Befort BL. Global food demand and the sustainable intensification of agriculture. Proc Natl Acad Sci U S A 2011; 108: 20260-4.

34. van der Sluis T, Pedroli B, Kristensen SBP, Lavinia Cosor G, Pavlis E. Changing land use intensity in Europe - Recent processes in selected case studies. Land Use Policy 2016; 57: 777-785.

35. Wu Y, Xi X, Tang X, Luo D, Gu B, Lam SK, et al. Policy distortions, farm size, and the overuse of agricultural chemicals in China. Proc Natl Acad Sci USA 2018; 115: 7010-7015.

36. You $\mathrm{H}, \mathrm{Hu} \mathrm{X}, \mathrm{Wu}$ Y. Farmland use intensity changes in response to rural transition in Zhejiang province, China. Land Use Policy 2018; 79: 350-361.

37. Zhang T, Ni J, Xie D. Assessment of the relationship between rural non-point source pollution and economic development in the Three Gorges Reservoir Area. Environ Sci Pollut Res Int 2016; 23: 8125-32.

38. Zhang Y, Long H, Li Y, Ge D, Tu S. How does off-farm work affect chemical fertilizer application? Evid. from China's mountainous and plain areas. Land Use Policy 2020; 99.

39. Zhu W, Wang R. Impact of farm size on intensity of pesticide use: Evidence from China. Science of The Total Environment 2020. 